\title{
The intermetallic semiconductor $h t$-IrGa3: a material in the in-transformation state
}

Raúl Cardoso-Gil ${ }^{1}$, Iryna Zelenina ${ }^{1}$, Quirin E. Stahl ${ }^{2}$, Matej Bobnar ${ }^{1, \dagger}$, Primož Koželj ${ }^{1}$, Mitja Krnel ${ }^{1}$, Ulrich Burkhardt $^{l}$, Igor Veremchuk ${ }^{1, \dagger \dagger}$, Paul Simon ${ }^{1}$, Wilder Carrillo-Cabrera ${ }^{l}$, Magnus Boström $^{1, \dagger \dagger \dagger}$, Yuri Grin ${ }^{1 *}$

${ }^{1}$ Max-Planck-Institut für Chemische Physik fester Stoffe, Nöthnitzer Str. 40, 01187 Dresden, Germany

${ }^{2}$ Institut für Festkörper- und Materialphysik TU Dresden, 01062 Dresden, Germany

*Corresponding Authors:

cardoso@cpfs.mpg.de (Raúl Cardoso-Gil)

grin@cpfs.mpg.de (Yuri Grin)

\section{Table of contents}

Table S1. Crystallographic data for $h t-\mathrm{IrGa}_{3}$ (sample 2)

Table S2. Atomic positions and displacement parameters $\left[\AA^{2}\right]$ for $h t-\operatorname{IrGa} 3$ (crystal 2)

Table S3. Lattice parameters and calculated density of $h t$-IrGa3 and $\operatorname{IrGa}_{-x} \mathrm{Zn}_{x}$ samples 
Table S1. Crystallographic data for $h t-\mathrm{IrGa}_{3}$ (sample 2). Lattice parameter are obtained from powder x-ray diffraction data, chemical composition from WDXS analysis.

\begin{tabular}{|c|c|c|}
\hline Composition & \multicolumn{2}{|l|}{$\mathrm{Ir}_{0.995(2)} \mathrm{Ga}_{3.000(2)}$} \\
\hline Molar mass & \multicolumn{2}{|l|}{401.38} \\
\hline Crystal color, shape & \multicolumn{2}{|c|}{ Gray, platelet like } \\
\hline Crystal dimensions $\left(\mathrm{mm}^{3}\right)$ & \multicolumn{2}{|c|}{$0.013 \times 0.020 \times 0.026$} \\
\hline Space group, $Z$ & \multicolumn{2}{|c|}{$P 4_{2} / m n m$ (no. 136$), 4$} \\
\hline Lattice parameters $(\AA)$ & \multicolumn{2}{|c|}{$a=6.4652(2) c=6.5676(2)$} \\
\hline$V\left(10^{6} \mathrm{pm}^{3}\right), \rho\left(\mathrm{g} \mathrm{cm}^{-3}\right)$ & \multicolumn{2}{|l|}{$274.52(3), 9.71$} \\
\hline Diffractometer, detector & \multicolumn{2}{|c|}{$\begin{array}{l}\text { Rigaku AFC7. CCD, } \\
\text { Saturn724+ }\end{array}$} \\
\hline Radiation & \multicolumn{2}{|c|}{$\operatorname{Mo} K \alpha(\lambda=0.71073 \AA)$} \\
\hline Exposures, steps & \multicolumn{2}{|l|}{$1370, \varphi=0.5^{\circ}$} \\
\hline Absorption correction & \multicolumn{2}{|c|}{ Multi-scan $\left(\mu=77.44 \mathrm{~mm}^{-1}\right)$} \\
\hline$T_{\min } / T_{\max }$ & \multicolumn{2}{|l|}{$0.134 / 0.365$} \\
\hline $2 \theta_{\max }$ & \multicolumn{2}{|l|}{$86.4^{\circ}$} \\
\hline$h k l$ range & \multicolumn{2}{|l|}{$-12<h<12$} \\
\hline & \multicolumn{2}{|l|}{$-4<k<10$} \\
\hline & \multicolumn{2}{|l|}{$-12<l<12$} \\
\hline Measured reflections & \multicolumn{2}{|l|}{3821} \\
\hline $\begin{array}{l}\text { Reflections used in } \\
\text { refinement }\end{array}$ & \multicolumn{2}{|l|}{2659} \\
\hline$R$ (int) & \multicolumn{2}{|l|}{0.031} \\
\hline Observation criteria & \multicolumn{2}{|c|}{$F(h k l)>4 \sigma F(h k l)$} \\
\hline Refinement & \multicolumn{2}{|c|}{ Full-matrix least-squares on $F^{2}$} \\
\hline & Ideal structure & Split model \\
\hline Parameters parameters & 15 & 36 \\
\hline$R(F), R w$ & $0.097,013$ & $0.061,0.064$ \\
\hline Goodness of fit & 0.06 & 1.00 \\
\hline$\Delta \rho_{\min }, \Delta \rho_{\max }\left(\mathrm{e} \AA^{-3}\right)$ & $-5.11,9.28$ & $-1.4,2.1$ \\
\hline
\end{tabular}


Table S2. Atomic positions and displacement parameters $\left[\AA^{2}\right]$ for $h t-\mathrm{IrGa}_{3}$ (crystal 2 ) in the $\mathrm{FeGa}_{3}$-type (upper panel) and in the split model (lower panel) .

\begin{tabular}{|c|c|c|c|c|c|c|c|c|c|}
\hline Atom & Site & $x / a$ & $y / b$ & $z / c$ & sof & $B_{\text {iso/eq }}$ & $\begin{array}{l}B_{11} \\
B_{12}\end{array}$ & $\begin{array}{l}B_{22} \\
B_{13}\end{array}$ & $\begin{array}{l}B_{33} \\
B_{23}\end{array}$ \\
\hline Ir & $4 f$ & $0.15862(8)$ & $\mathbf{x}$ & 0 & 1.00 & 1.051(9) & $\begin{array}{l}0.90(1) \\
0.14(4)\end{array}$ & $\begin{array}{c}B_{11} \\
0\end{array}$ & $\begin{array}{c}1.35(2) \\
0\end{array}$ \\
\hline Ga1 & $4 c$ & $1 / 2$ & $\mathbf{0}$ & $\mathbf{0}$ & 1.00 & $4.72(10)$ & $\begin{array}{l}2.58(14) \\
2.34(15)\end{array}$ & $\begin{array}{c}5.9(3) \\
0\end{array}$ & $\begin{array}{c}5.7(2) \\
0\end{array}$ \\
\hline Ga2 & $8 j$ & $0.8481(4)$ & $-x+1$ & $0.2422(11)$ & 1.00 & $4.55(6)$ & $\begin{array}{c}3.48(7) \\
-1.39(9)\end{array}$ & $\begin{array}{c}B_{11} \\
3.16(9)\end{array}$ & $\begin{array}{c}6.68(15) \\
-B_{13}\end{array}$ \\
\hline
\end{tabular}

\begin{tabular}{|c|c|c|c|c|c|c|}
\hline Atom & Site & $x / a$ & $y / b$ & $z / c$ & sof & $B_{\text {iso/eq }}$ \\
\hline Ir1 & $4 f$ & $0.1664(5)$ & 0.1664 & 0.5 & $0.29(7)$ & $0.73(5)$ \\
\hline Ir2 & & $0.1612(7)$ & 0.16121 & $0.5252(6)$ & $0.27(5)$ & $0.80(6)$ \\
\hline Ir3 & & $0.1515(3)$ & 0.15151 & 0.5 & $0.43(6)$ & $0.75(3)$ \\
\hline Ga11 & $4 c$ & $1 / 2$ & 0 & 0 & $0.41(1)$ & $1.30(5)$ \\
\hline Ga12 & & $0.4574(10)$ & $0.022(1)$ & $0.0430(8)$ & $0.44(2)$ & $1.39(7)$ \\
\hline Ga13 & & $0.554(3)$ & $0.128(3)$ & $0.407(3)$ & $0.13(1)$ & $1.24(9)$ \\
\hline Ga21 & $8 j$ & $0.911(2)$ & 0.08826 & $0.167(2)$ & $0.087(9)$ & $1.89(8)$ \\
\hline Ga22 & & $0.8467(4)$ & 0.15325 & $0.2627(5)$ & $0.43(2)$ & $1.48(4)$ \\
\hline Ga23 & & $0.8712(7)$ & 0.1287 & $0.2101(9)$ & $0.24(2)$ & $1.79(6)$ \\
\hline Ga24 & & $0.8223(7)$ & 0.17767 & $0.2967(8)$ & $0.23(3)$ & $1.63(7)$ \\
\hline Ga31 & $2 b$ & 0 & $\mathbf{0}$ & $1 / 2$ & $0.021(8)$ & $2.02(9)$ \\
\hline
\end{tabular}

an the split model, Ir1, Ir2 and Ir3 are split position of Ir in the ideal structure; Ga11 to G13 are split positions of Ga1 and Ga21 to Ga24 are split positions for $\mathrm{Ga} 2$. In the real structure model, Ir and Ga positions were refined in the isotropic approximation. ( $B_{\text {iso/eq }}$ defined as one third of the trace of the orthogonalized $B_{\mathrm{ij}}$ tensor). 
Table S3. Lattice parameters and calculated density of $h t-\mathrm{IrGa}_{3}$ and $\mathrm{IrGa}_{3-x} \mathrm{Zn}_{x}$ samples $\left(\mathrm{FeGa}_{3}\right.$ type, space group $\mathrm{P}_{2} / \mathrm{mnm}$ ) as-cast (upper values) and compacted material (lower values).

\begin{tabular}{|c|c|c|c|c|}
\hline Sample & $\begin{array}{l}\text { Nominal } \\
\text { composition }\end{array}$ & $a[\AA]$ & $c[\AA]$ & $d\left[\mathrm{~g} \mathrm{~cm}^{-3}\right]$ \\
\hline \multirow[t]{2}{*}{1} & $\mathrm{IrGa}_{3}$ & $6.4623(2)$ & $6.5688(2)$ & 9.72 \\
\hline & & $6.4617(2)$ & $6.5696(2)$ & 9.72 \\
\hline \multirow[t]{2}{*}{2} & $\mathrm{IrGa}_{3}$ & $6.4652(2)$ & $6.5676(2)$ & 9.71 \\
\hline & & $6.4657(1)$ & $6.5678(2)$ & 9.71 \\
\hline \multirow[t]{2}{*}{$3 a$} & $\operatorname{IrGa}_{2.92} \mathrm{Zn}_{0.08}$ & $6.4735(1)$ & $6.5790(1)$ & 9.54 \\
\hline & & $6.4832(2)$ & $6.5684(3)$ & 9.52 \\
\hline \multirow[t]{2}{*}{$3 b$} & $\operatorname{IrGa}_{2.84} \mathrm{Zn}_{0.16}$ & $6.4893(1)$ & $6.5708(2)$ & 9.62 \\
\hline & & $6.4906(2)$ & $6.5700(2)$ & 9.62 \\
\hline \multirow[t]{2}{*}{$3 c$} & $\operatorname{IrGa}_{2.76} \mathrm{Zn}_{0.24}$ & $6.5085(1)$ & $6.5635(2)$ & 9.56 \\
\hline & & $6.5097(3)$ & $6.5625(3)$ & 9.56 \\
\hline \multirow[t]{2}{*}{$3 d$} & $\operatorname{IrGa}_{2.68} \mathrm{Zn}_{0.32}$ & $6.5225(1)$ & $6.5537(2)$ & 9.53 \\
\hline & & $6.5239(1)$ & $6.5525(2)$ & 9.53 \\
\hline
\end{tabular}

